\title{
Pyrethrum ve Bacillus thuringiensis biyopestisitlerinin Pristiphora abietina (Christ, 1791) (Hymenoptera: Tenthredinidae) üzerindeki etkisi
}

\author{
The effect of Pyrethrum and Bacillus thuringiensis against Pristiphora abietina (Christ, 1791) (Hymenoptera: \\ Tenthredinidae)
}

Temel GÖKTÜRK

Artvin Çoruh Üniversitesi Orman Fakültesi Orman Mühendisliği Bölümü, Artvin, Türkiye

Eser Bilgisi / Article Info

Araştırma makalesi / Research article DOI: $10.17474 /$ artvinofd. 270322

Sorumlu yazar / Corresponding author Temel Göktürk

e-mail: temel.göktürk@artvin.edu.tr

Geliş tarihi / Received

29.11.2016

Düzeltme tarihi / Received in revised form

06.02.2017

Elektronik erişim / Online available

23.03.2017

Anahtar kelimeler:

Pristiphora abietina

Pyrethrum

Bacillus thuringiensis

Dipel DF

Keywords:

Pristiphora abietina

Pyrethrum

Bacillus thuringiensis

Dipel DF

\begin{abstract}
Özet
Son yıllarda Artvin Şavşat ladin ormanlarında zarara neden olan Küçük Ladin Arısına (Pristiphora abietina (Christ)) karşı tam bir mücadele stratejisi oluşturabilmek amacı ile 2014 yılında yapılan bu çalışmada; Pyrethrum ve Bacillus thuringiensis biyopestisitlerinin P. abietina üzerindeki etkisi araştırılarak uygulanabilirliği ortaya konulmaya çalışılmıştır. Laboratuvar şartlarında, $P$. abietina'nın larvalarına karşı farklı dozlarda Pyrethrum (Spruzit Neu) ve B. thuringiensis (Dipel DF) biyopestisitleri ile uygulamalar yapılarak sonuçlar Tek Yönlü Varyans Analizi ve Duncan Testi'ne göre değerlendirilmiştir. Larvalar için en etkili uygulamaların Dipel $100 \mathrm{gr} / 100 \mathrm{lt}$, Dipel $300 \mathrm{gr} / 100 \mathrm{lt}$ ve, Dipel $500 \mathrm{gr} / 100$ It uygulamaları olduğu tespit edilmiştir. Pyrethrum (Spruzit Neu)'un $150 \mathrm{ml} / 100 \mathrm{It}$ dozunda \% 71.7, $300 \mathrm{ml} / 100$ lt dozunda \% 81, $600 \mathrm{ml} / 100$ It dozunda \% 93.2, Dipel DF'in $100 \mathrm{gr} / 100$ It dozunda \% 91.5, $300 \mathrm{gr} / 100 \mathrm{It}$ dozunda \% 93.6, $500 \mathrm{gr} / 100 \mathrm{lt}$ dozunda ise \% 98.8 etkinlik oranı belirlenmiştir. Sonuç olarak, kullanılan her iki biyopestisitinde $P$. abietina'nın larvalarına karşı etkili olduğu tespit edilmiştir.
\end{abstract}

\begin{abstract}
This work has been done for the purpose of creating a complete struggle strategy against Pristiphora abietina (Christ), which is the cause of the spoil in Artvin Savsat spruce forests in the recent years and Pyrethrum and Bacillus thuringiensis biopesticides effects has been investigated and put into practice on the P. abietina. Under laboratory conditions, Pyrethrum (Spruzit Neu) and B. thuringiensis (Dipel DF) biopesticides were administered at different doses to the larvae of $P$. abietina and the results were evaluated according to the One Way Analysis of Variance and Duncan Test. The most effective applications for larval applications have been determined that Dipel $100 \mathrm{gr} / 100 \mathrm{lt}$, Dipel $300 \mathrm{gr} / 100$ It and Dipel $500 \mathrm{gr} / 100 \mathrm{It}$. The Pyrethrum (Spruzit Neu) efficiency ratio was determined $71.7 \%$ dose of $150 \mathrm{ml} / 100 \mathrm{lt}, 81 \%$ dose of $300 \mathrm{ml} / 100 \mathrm{lt}, 93.2 \%$ dose of $600 \mathrm{ml} / 100 \mathrm{lt}$ and Dipel DF was found to be $91.5 \%$ dose of $100 \mathrm{~g} / 100 \mathrm{lt}, 93.6 \%$ dose of $300 \mathrm{gr} / 100 \mathrm{lt}$ and $98.8 \%$ dose of $500 \mathrm{gr} / 100 \mathrm{lt}$, respectively. As a result, using of both biopesticides have been effective to against on larvae of $P$. abietina.
\end{abstract}

\section{Giriş}

Zararlı böcek türleri Ladin ormanlarında önemli ekonomik kayıplar meydana gelmesine sebep olmakta ve ormanların bu zararlı türlerden etkilenme şekli ve oranları farklılık gösterebilmektedir (Holusa ve ark. 2006; Taut ve ark. 2011). Artvin ladin ormanlarında 1966 yılından beri kabuk böceklerinin mevcutiyeti ilemilyonlarca ağaç zarar görmüştür. Uzun yıllar yürütülen mekanik ve biyolojik mücadele çalışmaları sonucu bu zararlı kabuk böceklerinin popülasyon yoğunluğu doğal denge sınırına çekilmiştir. Ancak, son yıllarda Pristiphora abietina'nın, Artvin Orman Bölge Müdürlüğü'nün Şavşat, Veliköy kısmındaki 1000 hektarlık ladin ormanında yoğun olmak üzere yaklaşık 16.582 hektarlık bir alanda zarar yaptığı belirtilmektedir (Aksu ve Göktürk 2008).

Pristophora cinsinin yaklaşık 50 türünden (Teager ve Blank, 2011) biri olan P. abietina, Avrupada ilk kez 1862 yılında tespit edilmiş ve tüm Avrupa ülkelerinde büyük sorun oluşturmuştur (Holusa ve Drapela 2006; Holusa ve Lubojacky 2007). Avrupa ve Rusya'da zararın hissedilir derecede çok olması kontrollerini de zorlaştırmıştır (Olenici ve Olenici 2005). Bu ibre zararlısı, özellikle doğal yayılış alanının dışındaki alanlardaki iğne yapraklı ormanlar için en tehlikeli zararlılardan biri olarak ifade edilmektedir (Holusa ve ark. 2005). P. abietina, larvalarının genç sürgünlerle yoğun bir şekilde beslenmesi sonucu direk olarak ibre dökümlerine, şekil 
bozukluklarına ve artım kayıplarına neden olması yanısıra zayıf düşen ağaçların kabuk böceklerinin saldırısına uğramasını kolaylaştırmasıyla türün zararı daha da artmaktadır.

$\mathrm{Bu}$ zararlı tür ile mücadelede bir çok yönteme başvurulmaktadır. Avrupada birçok bölgede zararlıya karşı sadece kimyasal ilaçlar kullanılırken (Gebert 1995; Olenici ve Olenici 2005), erginlerine karşı sarı yapışkan tuzaklar da kullanılmaktadır (Murhead 1991). Kuzey Amerika'da da erkek bireylerini cezbeden feromon tuzakları kullanılmıştırr (Anderbrant 1993).

Biyolojik insektisit olarak kullanılan Bacillus thuringiensis' in $P$. abietina larvalarına karşı etkili olduğu belirtmektedir (Manuel ve ark. 2008; Porcar ve ark. 2008). Avrupada yapılan mücadele çalışmalarında $P$. abietina'ya karşı biyolojik insektisit spinosad'ın etkili sonuçlar verdiği ve Entegre Zararlı Yönetiminde (IPM) kullanılabileceği belirtilmektedir (Valentin ve Gheorghe 2006). Ancak, bu türe karşı bir IPM stratejisi henüz oluşturulmamıştır (Holuša ve Drápela 2003). Artvin'de şimdiye kadar yürütülen mücadele çalışmalarında, $P$. abietina'nın yoğun zarar yaptığı alanda yürütülen arıcılık faaliyetlerinin ilaçlı mücadeleyi sınırlandırmasından dolayı deneme amaçlı Dimilin ODC 45 ve $B$. thrugiensis kullanılmasına ragmen (Aksu ve Göktürk 2008) populasyon yoğunluğu düşürülememiştir.
Yüzyıla yakın bir süre sentetik insektisitlerin tarım ve orman zararlılarına karşı kullanımı sonucunda, böceklerde dayanıklılık oluşmuş, canlılar toksik olarak etkilenmiş, bu da zararlılarla savaşta alternatif madde arayışını zorunlu kılmıştır. Bitkisel kökenli insektisitler ve biyoinsektisitler organik tarımla birlikte önem kazanmıştır. Dünyada en çok kullanılan bitkisel kökenli insektisitler olarak azadirachtin, pyrethrum, rotenone, nicotine, ryania, sabadilla, quassine ve bitkisel yağları (Güncan ve Durmuşoğlu 2004), biyoinsektisit olarak da Bacillus thuringiensis'i (Christensen 1990; Feitelson 1992; Martin ve Bonneau 2006; Cranshaw 2008; Shaukat ve ark. 2010) sayabiliriz.

$\mathrm{Bu}$ çalışmada, Pyrethrum ve $B$. thuringiensis biyopestisitlerinin $P$. abietina üzerindeki etkisi araştırılarak uygulanabilirliği ortaya konulmaya çalışılmıştır.

\section{MATERYAL VE YÖNTEM}

Çalışmanın ana materyalini Artvin Orman Bölge Müdürlüğü, Şavşat Orman İşletme Müdürlüğü Şavşat ve Veliköy Orman İşletme Şefliği ladin ormanlarından ladin uç sürgünlerinden toplanan $P$. abietina larvaları, taze ladin sürgünleri, tül kafesler, el pompası ve biyopestisitler oluşturmuştur. 2014 yılında gerçekleştirilen çalışmada, larvalara karşı yapılan uygulamalarla ilgili ilacın ticari adı ve kullanım dozu, kafessayısı, her bir kafese konulan larva sayısı bilgileri Tablo 1'de verilmiştir.

Tablo 1. Örnekleme düzeni

\begin{tabular}{|c|c|c|}
\hline İlacın Ticari Adı ve Kullanım Dozu & Kafes adedi & Larva adedi/Kafes \\
\hline Pyrethrum $\quad$ (Spruzit $\left.{ }^{\circ} \mathrm{Neu} 150 \mathrm{ml} / 100 \mathrm{lt}\right)$ & 11 & 20 \\
\hline (Spruzit ${ }^{\oplus}$ Neu $300 \mathrm{ml} / 100 \mathrm{lt}$ ) & 11 & 20 \\
\hline Pyrethrum $\quad$ (Spruzit $\left.^{\circ} \mathrm{Neu} 600 \mathrm{ml} / 100 \mathrm{lt}\right)$ & 11 & 20 \\
\hline Bacillus thuringiensis $\quad$ (DiPel ${ }^{\circ}$ DF BT 100gr/100lt) & 11 & 20 \\
\hline Bacillus thuringiensis $\quad$ (DiPel ${ }^{\circ}$ DF BT 300gr/100It) & 11 & 20 \\
\hline Bacillus thuringiensis $\quad$ (DiPel ${ }^{\circ}$ DF BT 500gr/100It) & 11 & 20 \\
\hline
\end{tabular}

Mayıs ayının ilk haftası $P$. abietina'nın 2. ve 3. dönem larvaları üzerinde beslenerek zarar yaptığı ladin dallarıyla birlikte alınarak Artvin Çoruh Üniversitesi Orman Fakültesi, Orman Mühendisliği Bölümünde bulunan Orman Entomolojisi laboratuarına getirilmiş ve $20 \times 20 \times 30 \mathrm{~cm}$ ebatlarında tel kafeslere her birini içerisinde 20 'şer adet larva olacak şekilde yerleştirilmiştir. Tel kafesler içerisine yerleştirlen ladin dallarının canlı kalması için su çekebilme özelliğindeki torflara batırılmış ve bu torflar nemli bırakılmıştır. Tablo 1 de verilen dozlarda üzerlerine püskürtme yöntemi ile biyopestisitler uygulanmıştır. Her uygulamada kontrol amacıyla seçilen 1 kafese safsu uygulaması gerçekleştirilmiştir. Biyopestisitlerin uygulanmasını takip eden her gün 12 saatte bir toplamda 10 kez kafesler kontrol edilerek canlı 
ve ölü larvalar sayılmıştır. Yapılan denemeler sonucu elde edilen sonuçlar istatistik analize tabi tutulmuştur.

\section{İstatistik Analiz}

Çalışmadaki verilerin değerlendirilmesinde SPSS 15.0 paket programı kullanılmıştır. Uygulanan ilaç ve dozlarının larvaların ölümü üzerindeki etkisini belirlemek amacıyla verilere Tek Yönlü Varyans Analizi (Oneway ANOVA) ve en etkili ilaç/ilaçları tespit etmek amacıyla da Duncan testi'nden faydalanılmıştır.

\section{BULGULAR VE TARTIŞMA}

Uygulamalarda tüm dozlar belli oranlarda etkili olmuştur. P. abietina'nın larvalarına karşı uygulanan altı farklı uygulamanın arasında anlamlı farklılıklar olduğu bulunmuştur (Tablo 2).

Tablo 2. Uygulanan ilaç ve dozların P. abietina'nın larvaları üzerindeki etkisini gösteren Tek Yönlü Varyans Analizi (Oneway ANOVA) sonuçları

\begin{tabular}{cccc}
\hline Gelişme Dönemi & Serbestlik derecesi (SD) & F değeri & Anlamlılık düzeyi (p) \\
\hline Larva & 5 & 29.612 & .0001 \\
\hline
\end{tabular}

P. abietina'nın larvalarına karşı yapılan biyopestisit uygulamalarından elde edilen verilerin analiz sonuçlarında; Pyrethrum'un $150 \mathrm{ml} / 100$ lt dozu ile 300 $\mathrm{ml} / 100$ It dozunun etkinliklerinin aynı olduğu görülmüştür. Analiz sonucunda Pyrethrum'un 600 ml/100 It dozu ile Difel'in $100 \mathrm{gr} / 100$ It dozunun etkinliği de benzer bulunmuştur. Uygulamanın istatistiksel analiz sonuçlarında Dipel'in tüm dozlarının etkinliklerinin benzer olduğu belirlenmiştir. $P$. abietina'nın larvaları için en etkili uygulamaların Bacillus thuringiensis (Dipel DF 100 gr/100 It), Dipel DF $300 \mathrm{gr} / 100$ lt, Dipel DF $500 \mathrm{gr} / 100$ It uygulamaları olduğu bulunmuştur (Tablo 3 ).

Tablo 3. Uygulanan ilaç ve dozların P. abietina'nın larvaları üzerindeki etkisini gösteren Homojenlik grup testi (Duncan Testi, $p=0.05)$ sonuçları

\begin{tabular}{|c|c|c|}
\hline & Ortalama & Standart Sapma \\
\hline (Spruzit ${ }^{\circ}$ Neu $150 \mathrm{ml} / 100 \mathrm{lt}$ ) & $14.9^{c}$ & \pm 1.2 \\
\hline (Spruzit ${ }^{\oplus}$ Neu $300 \mathrm{ml} / 100 \mathrm{lt}$ ) & $15.2^{\mathrm{c}}$ & \pm 1.3 \\
\hline (Spruzit ${ }^{\circ}$ Neu $600 \mathrm{ml} / 100 \mathrm{lt}$ ) & $18.2^{\mathrm{b}}$ & \pm 1.3 \\
\hline Bacillus thuringiensis $\quad$ (DiPel ${ }^{\circ}$ DF BT 100gr/100It) & $19.1^{\mathrm{ab}}$ & \pm 0.6 \\
\hline (DiPel ${ }^{\circ}$ DF BT 300gr/100lt) & $19.5^{\mathrm{a}}$ & \pm 0.5 \\
\hline (DiPel ${ }^{\circ}$ DF BT 500gr/100lt) & $19.6^{\mathrm{a}}$ & \pm 0.4 \\
\hline
\end{tabular}

Not: Aynı küçük harf bulunan ortalamalar arasında anlamlı farklılık yoktur $(p<0.05)$.

P. abietina'nın larvaları karşı kullanılan biyoinsektisitlerin etki oranları incelendiğinde, Pyrethrum (Spruzit Neu)'un $150 \mathrm{ml} / 100$ lt dozunda \% 71.7, $300 \mathrm{ml} / 100 \mathrm{lt}$ dozunda \% 81, $600 \mathrm{ml} / 100$ lt dozunda \% 93.2, Dipel DF'in $100 \mathrm{gr} / 100$ It dozunda \% 91.5, $300 \mathrm{gr} / 100$ lt dozunda \% 93.6, 500 gr/100 It dozunda ise \% 98.8 etkili olduğu belirlenmiştir (Şekil 1, 2). Uygulamalar sonrasında biyopestisitlerin en yüksek ölümlere 2. ve 4. günlerde neden olduğu görülmüştür.

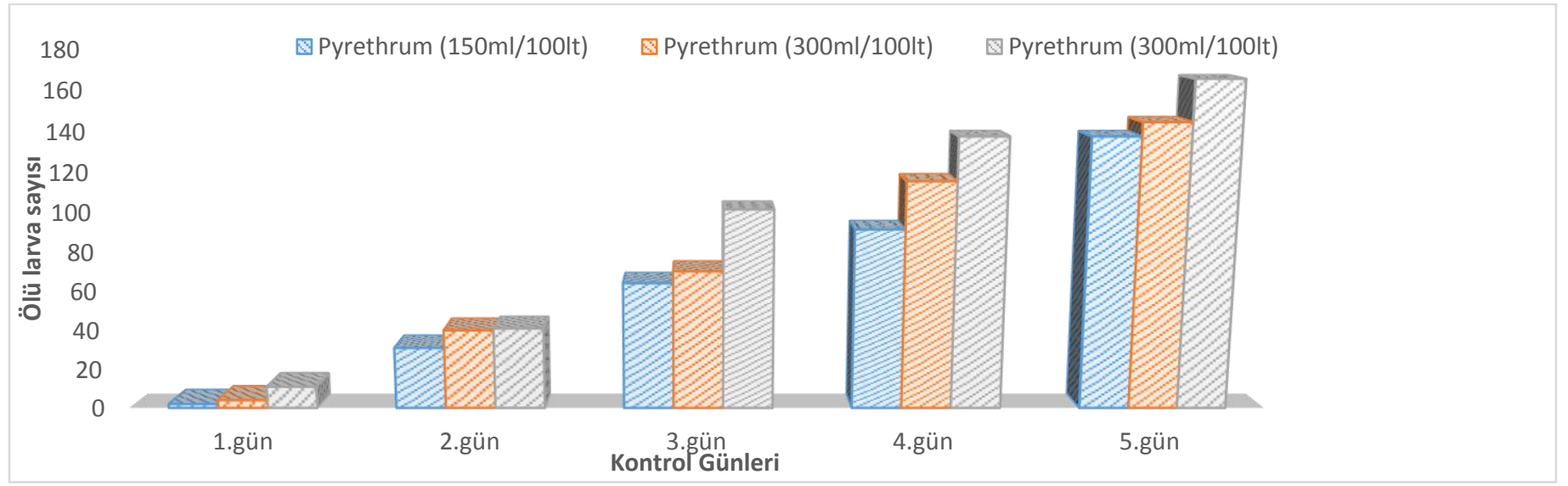

Şekil 1. Pyrethrum (Spruzit ${ }^{\circledR}$ Neu)’un farklı dozlarının larvalar üzerindeki etkinliği 


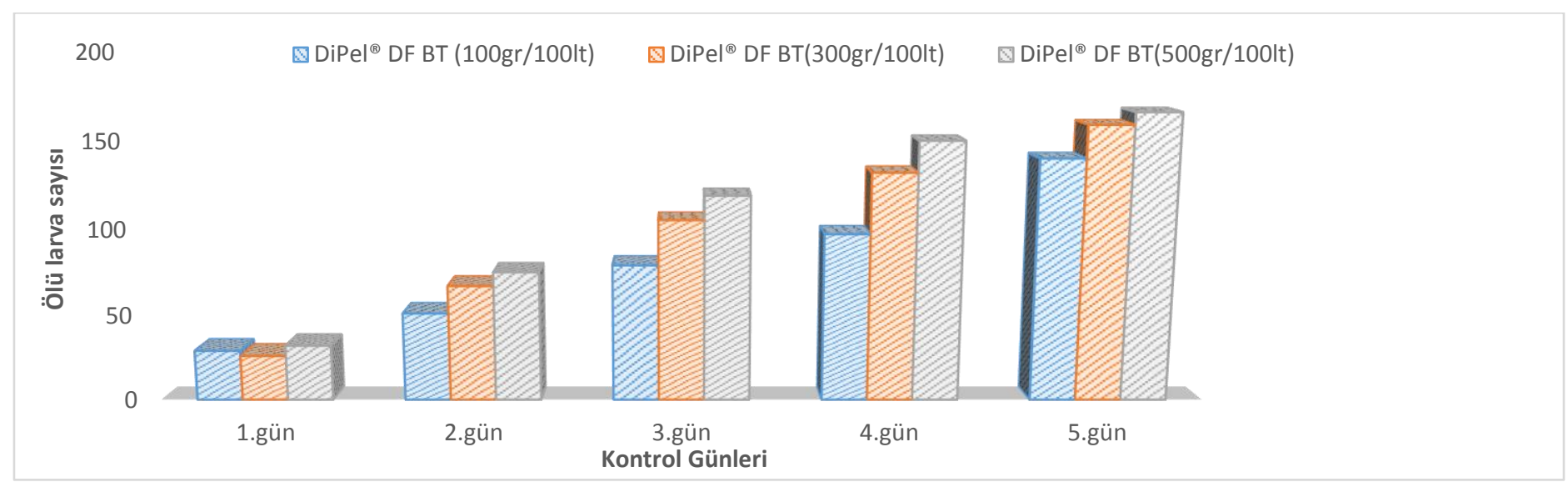

Şekil 2. Bacillus thuringiensis (DiPel ${ }^{\circledR}$ DF BT)'in farklı dozlarının larvalar üzerindeki etkinliği

Çalışmada elde edilen sonuçlara bakıldığında gerek Pyrethrum gerekse $B$. thuringiensis'in $P$. abietina larvalarını farklı oranlarda etkilediği tespit edilmiştir. Yurtdışında ve Türkiye'de $P$. abietina'ya karşı çeşitli mücadele çalışmaları yürütülmektedir. Egger (1989), $P$. abietina larvalarına karşı Malathion ULVC ve Dimilin $P H$ 60-40 preparatlarını uygulayarak \% 96,2 oranında popülasyon azalması gözlemlemiştir. Artvin'de ise bu zararlı türe karşı Dimilin ODC 45 (Aksu ve Göktürk 2008) ve $B$. thuringiensis kullanılmıştır. Deneme amaçlı 15 hektarılık bir alanda yapılan $B$. thuringiensis uygulaması sonucu $P$. abietina larvalarının \% 84'ünün öldüğü belirtilmiştir (Aksu ve ark. 2011). Yapılan çalışma sonuçlarına bakıldığında, $B$. thuringiensis preparatının $P$. abietina larvalarını \% 91.5 ile \% 98.8 oranında öldürdüğü belirlenmiştir.

\section{SONUÇ VE ÖNERILER}

Sentetik pestisitler, çevre ve insan sağlığı acısından riskler oluşturduğundan ve gerek tarım ve gerekse orman zararlılarına karşı kullanımının her geçen gün sınırlandırımasından dolayı zararlı böceklerle mücadelede alternatif savaş yöntemlerinin uygulanması zorunlu hale gelmiştir. Arıcılık ve hayvancılık yapılan alanlarda, sentetik pestisitlerin yerine bitkisel kökenli doğal pestisitler, biyoinsektisitler ve mikrobiyal preparatlar kullanılarak zararlılara karşı mücadele yapılabilmektedir.

Bölgede uzun zamandır kabuk böceklerine karşı yürütülen mücadele çalışmaları sonrası doğal denge sağlanmış olsa da $P$. abietina zararına maruz kalan ağaçların zayıf düşmesi kabuk böceği salgın tehlikesini olası kılacaktır.
Küçük Ladin Arısı P. abietina epidemi yaptığı Artvin Şavşat ormanlarının ciddi bir sorundur. 2007 yılından beri zararlının bölgedeki ağaçlarda oluşturduğu etki sonucu zayıf düşen ladin ağaçlarında yer yer kurumaların olduğu da görülmektedir. Bu zararlıya karşı önlem alınmadığı taktirde Artvin'in tamamına yayılma eğilimi gösterebileceği düşünülmektedir.

Bu çalışma sonucunda Pyrethrum (Spruzit ${ }^{\circ} \mathrm{Neu}$ ) ve $B$. thuringiensis (DiPel ${ }^{\circ}$ DF BT) biyopestisitinlerinin $P$. abietina'nın larvalarına karşı laboratuvar koşullarında etkili olduğu tespit edilmiştir. P. abietina'da larva dönemi 4 hafta sürdüğü için yürütülecek mücadele çalışmalarında bu durumun dikkate alınması önem arzetmektedir. Bu biyopestisitler $P$. abietina'nın larvalarına karşı arazi koşullarında da uygulanmalı ve başarılı sonuçlar alınması durumunda $P$. abietina'nın mücadele çalışmalarında kullanımının uygun olacağı sonucuna varılmıştır.

\section{KAYNAKLAR}

Aksu Y, Göktürk Çelik B (2008) Picea orientalis Ormanlarında Zarar Yapan Pristiphora abietina (Christ) (Hymenoptera: Tenthredinidae)'nin Biyolojisi, Morfolojisi ve Mücadelesi Üzerine Yapılan Araştırma, Orman Mühendisleri Dergisi. 45, sayı: 110, 11, 12, s. 35-39.

Aksu, Y, Göktürk ÇG, Morkan L, Subaşı E (2011) Picea orientalis'lerde Zarar Yapan Pristiphora abietina (Christ) (Hymenoptera: Tenthredinidae)'nın Zararı ve Mücadelesi. Türkiye I. Orman Entomolojisi ve Patolojisi Sempozyumu, 23 - 25 Kasım Antalya, s. 298-301.

Anderbrant O (1993) Pheromone Biology of Sawflies. In: Wagner M.R., Raffa K.F. (eds.), Sawfly Life History Adaptations to Woody Plants. San Diego, London, Academic Press Inc.: 119-154.

Christensen KP (1990) Dipel Technical Material (Bacillus thuringiensis var. kurstaki): Infectivity and Pathogenicity to Rainbow Trout 
(Oncorhynchus mykiss) during a 32-Day Static Renewal Test. Wareham, Massachusetts, Springborn Laboratories Inc., pp 1-57

Cranshaw WS (2008) Bacillus thuringiensis. Colorado State University Extension. No. 5. 556.4pp.

Egger VA (1989) On the Outbreak and Aviochemic Control of Pristiphora abietina (Christ.) (Hym.,Tenthredinidae) Anz. Scfiadlingskde., Pflanzenschutz, Uımveltschutz 62, 127- 132.

Feitelson JS, Payne J, Kim L (1992) Bacillus thuringiensis: Insects and Beyond. Bio/Technology 10, 271-275.

Gebert AK (1995) Neue Erkenntnisse zur Kleinen Fichtenblattwespe (Pristiphora abietina Christ). LWF Actuell 2 www.Iwf.bayern.de/Iwfaktuell/LWFaktuell2-95/fiblatr.html Güncan A, Durmuşoğlu E (2004) Bitkisel Kökenli Doğal Insektisitler Üzerine Bir Değerlendirme. Hasad dergisi, 20 (233): 26-32.

Holuša J, Drápela K (2003) Integrated Management of Little Spruce Sawfly (Pristiphora abietina): Design Pattern in McManus, M.-L. \& Liebhold, A. M. (eds.) Proceedings Ecology, Survey and Management of Forest Insects. USDA Forest Service, 16-24.

Holuša J, Drápelak K (2006) Yellow Sticky Boards: A Possible Way of Monitoring Little Spruce Sawfly (Pristiphora abietina) (Hymenoptera: Tenthredinidae), Journal of Forest Science, 52 (1): 13-21.

Holuša J, Liška J, Kapitola P, Pesková V, Soukup F (2006) The Phytopathological and Entomological Aspects of The Health of Mountain Norway Spruce Stands in The Czech Republic, Current Problems of Forest Protection in Spruce Stands under Conversion. Papersfroma scientic workshop held at Ustron'-Jaszowiec, Poland, 13-14 October, 1: 85-92.

Holusa J, Liska J, Kapitola P, Peskova V, Soukup F (2005) Aspekt Fitopatologicznyi Entomologiczny Zdrowotnosci Swierczyn Gorskichna Terenie Republiki Czeskiej, Lesne Prace Badawcze, 2: 133-138.

Holusa J, Lubojacky J (2007) Correlation Between. Flight Activity of Sawflies Pristiphora abietina, $P$. saxesenii, $P$. gerula and $P$. leucopodia (Hymenoptera: Tenthredinidae) and Spruce (Picea abies) Bud Breaking in Eastern Czech Republic, Journal of Forest Science, 53: 69-73

Manuel P, Francesc G, Axel G, Antonio GP, Inmaculada S, Roland S (2008) Integrated Management of Little Spruce Sawfly (Pristiphora abietina). Design Pattern Biological Control 45: 427-432.

Martin JC, Bonneau X (2006) Bacillus thuringiensis, 30 Years of Control of Cluster Caterpillars. Phytoma. 590: 4-7.

Murhead-Thompson RC (1991) Trap Responses of Flying Insects. The Influence of Trap Design on Capture Efficiency. Academic press, London, San Diego, New York, Boston, Sydney, Tokyo, Toronto, $288 \mathrm{pp}$.

Olenici N, Olenici V (2005) Pristiphora abietina (Christ) (Hymenoptera, Tenthredinidae) un Daunator Important Al Molidului Din Afara Arealului Natural de Vegetatie. Revista padurilor, 120 (1): 3-13.

Porcar M, Gómez F, Gruppe A, Gómez-Pajuelo A, Segura I, Schrödere $R$ (2008) Hymenopteran specificity of Bacillus thuringiensis strain PS86Q3. Biol Control 45 (3): 427-432

Shaukat A, Yusuf Z, Ghulam Muhammad A, Farhat N (2010) Bacillus thuringiensis and Its Application in Agriculture, African Journal of Biotechnology Vol. 9 (14), pp. 2022-2031.

Taeger A, Blank SM (2011) ECatSym - Electronic World Catalog of Symphyta (Insecta, Hymenoptera). Program Version 3.9, Data Version 38 (07.12.2011). - Digital Entomological Information, Müncheberg.

Tâut I, Simonca V, Holonec L (2011) Detection and Prognosis Defoliators Tortrix viridana and Pristiphora abietina in The Northwestern Transylvania Forests, Bulletin U.S.A.M.V. ClujNapoca, Horticulture, 68 (1): 469-473.

Valentin B, Gheorghe P (2006) Bioecology and Control Researches Concerning The Little Spruce Sawfly Pristiphora abietina (Christ) (Hymenoptera; Tenthredinidae) Analele Ştiinţifice ale Universităţii AL. I. CUZA" Iaşi, s. Biologie animală, Tom LII, 131-136. 